\title{
A Review on Energy-Saving Optimization Methods for Robotic and Automatic Systems
}

\author{
Giovanni Carabin (1), Erich Wehrle (1D) and Renato Vidoni * \\ Faculty of Science and Technology, Free University of Bozen-Bolzano, 39100 Bolzano, Italy; \\ Giovanni.Carabin@natec.unibz.it (G.C.); Erich.Wehrle@unibz.it (E.W.) \\ * Correspondence: Renato.Vidoni@unibz.it; Tel: +39-0471-017203
}

Received: 20 October 2017; Accepted: 4 December 2017; Published: 7 December 2017

\begin{abstract}
In the last decades, increasing energy prices and growing environmental awareness have driven engineers and scientists to find new solutions for reducing energy consumption in manufacturing. Although many processes of a high energy consumption (e.g., chemical, heating, etc.) are considered to have reached high levels of efficiency, this is not the case for many other industrial manufacturing activities. Indeed, this is the case for robotic and automatic systems, for which, in the past, the minimization of energy demand was not considered a design objective. The proper design and operation of industrial robots and automation systems represent a great opportunity for reducing energy consumption in the industry, for example, by the substitution with more efficient systems and the energy optimization of operation. This review paper classifies and analyses several methodologies and technologies that have been developed with the aim of providing a reference of existing methods, techniques and technologies for enhancing the energy performance of industrial robotic and mechatronic systems. Hardware and software methods, including several subcategories, are considered and compared, and emerging ideas and possible future perspectives are discussed.
\end{abstract}

Keywords: energy savings; robotic systems; system enhancement; trajectory optimization; operations scheduling; energy recovery; natural motion

\section{Introduction}

The aim of this paper is to provide a comprehensive review and, therefore, to provide a reference of existing methods, techniques and technologies for enhancing the energy performance of industrial robotic and mechatronic systems, in particular, concerning electrical energy consumption. This documentation is intended to be a source of information for both engineers and scientists working on new solutions for a higher efficiency of industrial robotic and mechatronic systems. The different options currently available are categorized here into hardware and software.

The former exploits the availability of new materials, allowing for a lighter design while still providing the necessary structural-mechanical properties. Hardware solutions also include technologies for implementing new kinds of driving systems as well as energy recovery and distribution strategies. These allow for new scenarios of reduced energy consumption and therefore less environmental impact.

The software solution starts from the idea that energy consumption in industrial manufacturing facilities predominantly results from the operation and control of electrical drives in automated manufacturing processes such as assembly and packaging. Machines and robots are often operated dynamically to maximize production outputs (i.e., minimization of time), thus causing both high energy losses at high velocities as well as energy surpluses when decelerating. In addition, many axis-movement tasks are followed by idle times associated with a loss of productivity. Hence, methods and techniques for the minimization of the energy used have became important and worthy of investigation. 
Finally, a third category can be identified, the mixed approach, in which both the hardware and the software modifications of the mechatronic system are considered together.

The paper is organized as follows: In Section 2, the different hardware solutions for enhancing the energy performances are discussed. In Section 3, the main software techniques for minimizing the energy used via motion-planning modification are reviewed. In Section 4, the most recent developments, in which both hardware and software approaches are considered in concert, are presented. Finally, Section 5 contains the conclusion, in which future directions to further increase the energy efficiency of mechatronic and robotic systems are discussed.

\section{Hardware: Energy-Saving Design and Technologies}

A variety of approaches have been proposed in literature to achieve the reduction of energy consumption by concentrating on the hardware aspect. The hardware approach achieves this objective through the optimal design of new systems as well as by considering the substitution or addition of energy-efficient components of existing systems. See Table 1 for an overview [1-18].

Hardware improvement of robotics for energy efficiency can be further divided into the following three subcategories:

1. Robot type: The selection of more energy-efficient mechatronic and robotic systems available for a given application $[4,5,11,19]$.

2. Hardware replacement: The re-design or substitution of components with more efficient components (i.e., more-efficient or lighter components, or both) $[1,2,6,7,10,12,16,18]$.

3. Hardware addition: The addition of components for storing and recovering energy [20-36].

These are introduced below.

\subsection{Robot Type}

Through the proper choice of available automation system for a given application, an energy enhancement can be achieved, meanwhile keeping the productivity $[4,5,11,19]$. In [4], a comparison between parallel and serial manipulator configurations of a similar workspace and payload is carried out. The parallel configuration is shown on average to be more energy efficient in horizontal motion. However, it is less energy efficient in vertical movement. In [5], an under-actuated serial robot with three degrees of freedom (DOFs) is presented and compared with respect to industrial conventional robots (e.g., linear-axis system, or industrial robotic system) to operate a pick-and-place operation.

Further, the adoption of redundant manipulators is considered as a possible solution if an energy consumption reduction is needed. In [11,19], the effect of the kinematic redundancy insertion on the energy demand of parallel manipulators is considered. In this case, the redundantly actuated system can distribute arbitrary actuating torques in a certain combination; thus that which is more efficient can be chosen. Similarly, in [19], a comparison between a 3 Revolute joints (3RRR), non-redundant, planar, parallel manipulator and its kinematically redundant versions is performed, demonstrating that an energy-consumption reduction can be obtained in the second case. In [11], a robotic system based on a parallel mechanism with redundant actuation is evaluated, showing possibilities to reduce electric power loss, peak torque of actuating joints and friction loss. 
Table 1. Hardware: Energy-saving (re-)design and technologies literature.

\begin{tabular}{|c|c|c|c|c|c|c|}
\hline Reference & Year & Document Type & Category & Subcategory & & Case Study \\
\hline$[4]$ & 2001 & Conference paper & Robot type & - & - & 3-DoFs parallel manipulator and serial manipulator \\
\hline [5] & 2014 & Conference paper & Robot type & - & - & Underactuated serial kinematics \\
\hline [11] & 2015 & Journal paper & Robot type & - & - & Parallel manipulator with redundant actuation \\
\hline [19] & 2015 & Conference paper & Robot type & - & - & Parallel manipulator with redundant actuation (3RRR) \\
\hline [15] & 2010 & Review & Replacement & Electric actuator & - & Generic mechatronic system \\
\hline [17] & 2009 & Journal paper & Replacement & Pneumatic & - & Generic pneumatic system \\
\hline [7] & 2002 & Conference paper & Replacement & Reduction of components weight & Lighter arms & Redundant serial robot (7 DoFs) \\
\hline [18] & 2016 & Conference paper & Replacement & Reduction of components weight & Lighter arms & Serial robot (5 DoFs) \\
\hline [6] & 2008 & Journal paper & Replacement & Reduction of components weight & Lighter joints & Redundant serial robot (7 DoFs) \\
\hline$[2]$ & 2016 & Conference paper & Replacement & Moving the actuators at the base & - & Serial robot (3 DoFs) \\
\hline$[10]$ & 2015 & Conference paper & Replacement & Moving the actuators at the base & - & Elbow joint (1 DoF) wrist joint (3 DoFs) \\
\hline [12] & 1993 & Journal paper & Replacement & Moving the actuators at the base & - & Redundant serial robot (7 DoFs) \\
\hline [16] & 2012 & Journal paper & Replacement & Moving the actuators at the base & - & Underactuated serial kinematics \\
\hline [20] & 2015 & Conference paper & Addition & Energy-storing devices & Flywheel & Serial robot (6 DoFs)- ABB IRB 140 \\
\hline [21] & 2011 & Conference paper & Addition & Energy-storing devices & Flywheel & Serial robot (3 DoFs)-crane \\
\hline$[22]$ & 2012 & Conference paper & Addition & Energy-storing devices & Hydraulic & Generic hydraulic system \\
\hline [23] & 2014 & Journal paper & Addition & Energy-storing devices & Hydraulic & Generic hydraulic system \\
\hline [26] & 2013 & Conference paper & Addition & Energy-storing devices & Several & Generic mechatronic system \\
\hline [27] & 2011 & Conference paper & Addition & Energy-storing devices & Supercapacitor & Mechatronic system (1 DoF)-elevator \\
\hline [28] & 1997 & Conference paper & Addition & DC-bus sharing & $1-$ & Mechatronic system-drives \\
\hline [29] & 2011 & Conference paper & Addition & DC-bus sharing & EnergyTeam & Generic mechatronic system-robot \\
\hline [30] & 2012 & Conference paper & Addition & DC-bus sharing & Single capacitor & Generic mechatronic system-robot \\
\hline [31] & 2013 & Conference paper & Addition & DC-bus sharing & Single capacitor & Generic mechatronic system-robot \\
\hline [32] & 2013 & Journal paper & Addition & DC-bus sharing & Single capacitor & Generic mechatronic system-robot \\
\hline
\end{tabular}




\subsection{Hardware Replacement}

The second of the considered strategies is the lighter design of components to reduce the moving masses; this means reducing the weight and inertia of arms. In this manner, the driving torque can be reduced, in turn using less energy and leading to an easier control $[1,2,6,7,10,12,16,18]$. In [7], arms made of an ultralight carbon-fibre-reinforced polymer are studied, while in [18], an optimal design of a serial-link robotic manipulator is evaluated. In the latter, the aim is to find the arms' optimum diameter as well as the optimum dimensions of lightening holes in order to minimize the masses without detrimentally affecting the structural-mechanical behavior. Lighter components can be installed as in $[1,6]$, in which special lightweight rotary joints are designed as the result of an optimization process.

Following the well-known idea of reducing the moving masses by moving the actuator to the robot base $[2,10,12,16]$, different solutions have been proposed for serial-link manipulators. In these works, the opportunity of installing the motor near or on the chassis is often achieved by transferring the motion via pulleys and timing belts. In [2], a planar redundant manipulator with three degrees of freedom is presented, in which the motion is transferred through aluminium pulleys, bearings, and timing belts. In [10,12], a lightweight tension amplifying mechanism is used: a joint is moved by pushing and pulling two cords connected on it, on diametrically opposed positions. In this way, the weight of the arms is reduced, as the motors are fixed on the chassis. Beyond this, it increases the joint stiffness, which is essential for a precise and rapid manipulation. In [16], a mechanical design of a three-dimensional, planar, redundant manipulator in which only three motors, mounted near the base, are needed to control a system of any number of degrees of freedom is presented. In more detail, the third motor actuates all except the first two joints as a result of the connection through shafts and gears between links.

Focusing the attention on the driving system, for example, the motor and transmission, different options can be implemented to increase energy efficiency. Saidur [15] presents a detailed analysis on electric-motor energy losses and on strategies to increase the efficiency. These are mostly based on the design of a more efficient motor (e.g., increasing the cross-section of the coil, and the design of a low-loss magnetic core). In $[8,9,14]$, the optimal efficiency design of a gear train, in terms of the optimal gear ratio taking into account the Coulomb and viscous friction, is presented. In [17], an application for a pneumatic asymmetric cylinder actuator is presented. In this application, it is possible to save energy by connecting the two chambers through a bypass valve during the constant-speed phase. Under this condition, an air pressure difference exists between the two chambers, as forces are equilibrated and the presence of the rod reduces one of the piston areas.

\subsection{Hardware Addition: Energy Storing and Recovering}

One of the most recent techniques adopted to reduce the energy expenditure of mechatronic and robotic systems is the addition of energy-storing and -recovering devices. The main idea behind these devices is to harvest the energy during the braking phase-which is usually wasted-store it, and provide it back to the system when necessary [20-32]. Two different types are considered and analyzed: (1) the type of energy-storing devices used, and (2) energy-sharing via the interaction between actuators exchanging energy in a multi-actuator system.

\subsubsection{Energy-Storing-Devices Type}

The energy-storing-devices type includes the components that allow the recovering and storing of energy to use when needed. According to [26], these kinetic-energy recovery systems (KERS) can be subdivided into the following:

1. Mechanical KERS (e.g., flywheels).

2. Electric KERS (e.g., chemical batteries, capacitors, and supercapacitors).

3. Hydraulic KERS (e.g., hydro-pneumatic accumulator). 
4. Hydro-electric KERS (e.g., a hydraulic motor coupled with an electric generator).

In [26], a comparison between the different types of energy-recovery systems is made, considering aspects such as voltage instability, working temperature range, efficiency and cost. According to the authors, the flywheel results as the best KERS system in terms of voltage stability, temperature range and efficiency.

In $[20,21]$, a flywheel is added to a mechatronic system in order to store energy: in this case, the flywheel is considered as an additional axis of the system. Gale et al. [20] simulated an anthropomorphic manipulator programmed to execute a number of typical movements generally used in manufacturing. The results show that a flywheel-based energy-storage system is fully compatible with the manipulator controller hardware, and it is able to achieve a reduction in power consumption. $\mathrm{Xu}$ et al. [21] analyzed the case of a three-degrees-of-freedom, rubber-tyred gantry crane robot provided with a surface-mounted permanent magnet synchronous motor (PMSM) and an integrated flywheel spinning at high speed. They demonstrated that the method can be used to perfectly meet the peak energy requirements of the crane during both acceleration and regenerative braking.

Pneumatic driven systems (e.g., grippers and actuators) are widely used in mechatronic systems given their simplicity and reliability. However, their efficiency is lower with respect to corresponding hydraulic and electrical technologies [24,25]. Thus, interesting results could be expected by applying an energy-reducing method. In [24], a hybrid pneumatic-electrical system that recovers exhaust air energy from pneumatic actuator outlets to generate electricity through a scroll-type air motor is proposed. In [25], an energy recovery scheme is studied that reuses the pressurized air released by retracting pneumatic soft actuators instead of venting to the atmosphere. Simulation results show that a suitable air accumulator allows savings of about $20 \%$ of the energy consumption with an acceptable residual pressure [25].

Hydraulically actuated systems have also been used. In [22], a closed-loop hydrostatic transmission equipped with a hydraulic energy-regenerative system is presented. The hydraulic accumulator is used as the energy-storage system without a reversal of the fluid flow. In [23], the authors substitute the hydraulic compensator, a device that allows governing the pressure drops over the control orifices in a plant, with a regenerative device: a hydraulic motor coupled with an electric generator and an electrical capacitor to store recovered energy. In this case, the pressure compensation is realized by adapting the electromagnetic torque of the generator to the load.

\subsubsection{Energy-Sharing Devices}

Energy-sharing devices base their working principle on the sharing of braking energy on a common network for driving other (non-braking) actuators. The most common way to implement such an idea is the DC-bus sharing system. Today, some commercial robot controllers allow for recuperating energy when a motor brake occurs (i.e., when one axis is braking, others that accelerate can use that energy). In most cases, if several axes brake at the same time, the available energy exceeds the installed DC-bus capacitance. Thus, the energy is dissipated on a balancing or chopper resistor [30]. By properly designing and adding the number of storing devices, this energy can be almost fully recovered and then shared. Either a single centralized or multiple decentralized energy buffers and rectifiers are to be chosen, as well as the proper line and the type of storing buffer (e.g., capacitor, flywheel, or pneumatic). In [29], the single capacitor energy buffer approach and EnergyTeam (i.e., a multi-star DC-bus connection of several industrial controllers with their own rectifier module) are analyzed and compared in terms of cost efficiency. The addition and installation of capacitor banks is relatively simple but is not so economical. The EnergyTeam is a promising solution, but it requires a robot controller able to receive external DC power. In [30-32], the case of a single centralized capacitor that is shared between different DC subgrids (i.e., a subgrid is composed by several drive systems), each one with its own rectifier, is considered. In these papers, a method to avoid the synchronization between every rectifier's subgrids is proposed. 
In [28], the advantages of a shared/common-bus operation of AC drives are shown, which include cost reduction, reduced space requirements and improved reliability. In [32], an investigation is presented on how shared DC-bus systems with a common storage capacitor affect the exploitation of regenerated energy from the electric drives of an industrial robot. Simulations to obtain specific efficiency indicators of the system with respect to the number of shared DC buses were performed, showing that, with a sufficiently high capacity, the regeneration energy of several systems can be efficiently utilized. Moreover, it is demonstrated that the number of robots does not affect the amount of energy saved.

\section{Software: Enhancement of the Motion Planning Phase}

The software approach relies on the modification and optimization of the motion planning phase of a mechatronic system. The first step of the general workflow of the software approach is the definition of a suitable system model: this is exploited to perform simulations and the analysis of different strategies, through the evaluation of the energy consumption and the dynamic behavior of the mechatronic system [37]. In literature, different approaches are used to build the model; it can be defined through a mathematical formulation and by considering only the main important factors (i.e., basic mathematical model) [38-70], or it is possible to also take into account secondary terms that increase the results' precision but on the other hand need more computational efforts (i.e., extended mathematical model) [71-77]. The availability of software simulation tools allows thus the simple implementation of complex extended models [78-81] that can describe several physical domains [37,82-85]. These allow for the integration of several simulation approaches that are evaluated separately: control, kinematic, dynamic, physical behavior, and discrete event simulations [83]. The workflow proceeds with the assignment of the system parameters, such as links' lengths, masses, actuator electrical parameters, and so forth. These can be retrieved by datasheets, the CAD model, estimation, or eventually direct or indirect measurements of the system. Then, a comparative analysis with a real system is generally performed to validate the overall dynamic model and possibly to improve it [37]. The system model is thus then ready to be exploited for testing the software optimization strategies.

Different solutions relying on the software approach have been published; see Table 2. All of these can be divided into two main subcategories:

1. Trajectory optimization: A modification of the path or the motion profile, or both, are performed.

2. Operation scheduling: Re-scheduling of subsequent movements and operations is considered. 
Table 2. Literature on energy-saving software.

\begin{tabular}{|c|c|c|c|c|c|}
\hline Reference & Year & Document Type & Category & Subcategory & Case Study \\
\hline [71] & 2014 & Journal paper & Trajectroy optimization & PTP-direct & Mechatronic system (1 DoF)-elevator \\
\hline$[72]$ & 2014 & Conference paper & Trajectroy optimization & PTP-direct & Generic mechatronic system (1 DoF) \\
\hline [37] & 2010 & Conference paper & Trajectroy optimization & PTP-direct & Serial robot (6 DoFs) \\
\hline$[84]$ & 2010 & Journal paper & Trajectroy optimization & PTP-direct & Serial robot (6 DoFs) \\
\hline [85] & 2014 & Journal paper & Trajectroy optimization & PTP-direct & Serial robot (6 DoFs) \\
\hline [73] & 2016 & Journal paper & Trajectroy optimization & PTP-direct & Mechatronic system (1 DoF)-constant inertia \\
\hline [74] & 2012 & Journal paper & Trajectroy optimization & PTP-direct & Storage and retrieval vehicle (2 DoFs) \\
\hline [75] & 2013 & Journal paper & Trajectroy optimization & PTP-direct & Storage and retrieval vehicle (2 DoFs) \\
\hline [38] & 2013 & Conference paper & Trajectroy optimization & PTP-inverse & Serial robot (1 DoF) mounted on a flexible base \\
\hline [39] & 2016 & Journal paper & Trajectroy optimization & PTP-inverse & Serial robot (1 DoF) mounted on a flexible base \\
\hline [40] & 2016 & Journal paper & Trajectroy optimization & PTP-inverse & Redundant serial robot (3 DoFs) \\
\hline [41] & 2010 & Conference paper & Trajectroy optimization & PTP-inverse & Serial robot $(6 \mathrm{DoFs})$ \\
\hline [57] & 2015 & Conference paper & Trajectroy optimization & PTP-inverse & Mechatronic system (1 DoF)-linear axis \\
\hline [42] & 1996 & Conference paper & Trajectroy optimization & PTP-inverse & Serial robot $(6 \mathrm{DoFs})$ \\
\hline [43] & 2011 & Journal paper & Trajectroy optimization & PTP-inverse & Underactuated serial kinematics \\
\hline [44] & 2011 & Conference paper & Trajectroy optimization & PTP-inverse & Underactuated serial kinematics \\
\hline [46] & 2014 & Journal paper & Trajectroy optimization & PTP-inverse & Underactuated serial kinematics \\
\hline [49] & 2013 & Conference paper & Trajectroy optimization & PTP-inverse & Generic mechatronic system (2 DoFs) \\
\hline [48] & 2012 & Conference paper & Trajectroy optimization & PTP-inverse & Serial robot $(6 \mathrm{DoFs})$ \\
\hline [50] & 2012 & Conference paper & Trajectroy optimization & PTP-inverse & Generic mechatronic system (1 DoF) \\
\hline [51] & 2011 & Conference paper & Trajectroy optimization & PTP-inverse & Mechatronic system (1 DoF)-positoning table \\
\hline [45] & 2012 & Conference paper & Trajectroy optimization & PTP-inverse & Mechatronic system (1 DoF)-toggle mechanism \\
\hline [47] & 2014 & Journal paper & Trajectroy optimization & PTP-inverse & Mechatronic system (1 DoF)-toggle mechanism \\
\hline [52] & 2011 & Conference paper & Trajectroy optimization & PTP-inverse & Mechatronic system (1 DoF)-positioning table \\
\hline [53] & 2012 & Journal paper & Trajectroy optimization & PTP-inverse & Mechatronic system (1 DoF)-toggle mechanism \\
\hline [86] & 2011 & Conference paper & Trajectroy optimization & PTP-inverse & Generic mechatronic system (2 DoFs) \\
\hline [54] & 2006 & Conference paper & Trajectroy optimization & PTP-inverse & Redundant serial robot (3 DoFs) \\
\hline [55] & 1996 & Journal paper & Trajectroy optimization & PTP-inverse & Generic mechatronic system (1 DoF) \\
\hline [56] & 2002 & Conference paper & Trajectroy optimization & PTP-inverse & Generic mechatronic system (1 DoF) \\
\hline [58] & 2015 & Journal paper & Trajectroy optimization & PTP-inverse & Generic mechatronic system (1 DoF) \\
\hline [59] & 2004 & Conference paper & Trajectroy optimization & PTP-inverse & Flexible links robot (2 links) \\
\hline$[60]$ & 2017 & Conference paper & Trajectroy optimization & PTP-inverse & Redundant serial robot (4 DoFs) \\
\hline
\end{tabular}


Table 2. Cont.

\begin{tabular}{|c|c|c|c|c|c|}
\hline Reference & Year & Document Type & Category & Subcategory & Case Study \\
\hline [71] & 2016 & Conference paper & Trajectroy optimization & Multi-point & Serial robot (6 DoFs) \\
\hline [80] & 2016 & Conference paper & Trajectroy optimization & Multi-point & Serial robot (6 DoFs) \\
\hline [68] & 1997 & Conference paper & Trajectroy optimization & Multi-point & Redundant serial robot (3 DoFs) \\
\hline [81] & 2014 & Conference paper & Trajectroy optimization & Multi-point & Serial robot $(6 \mathrm{DoFs})$ \\
\hline [69] & 2014 & Conference paper & Trajectroy optimization & Multi-point & Serial robot (6 DoFs) \\
\hline [70] & 2011 & Conference paper & Trajectroy optimization & Multi-point & Serial robot (2 DoFs) \\
\hline [77] & 2014 & Journal paper & Operation scheduling & Time scaling & Serial robot (6 DoFs) \\
\hline [76] & 2012 & Conference paper & Operation scheduling & Time scaling & Serial robot (6 DoFs) \\
\hline [87] & 2006 & Journal paper & Operation scheduling & Time scaling & Serial robot $(2 \mathrm{DoFs})$ \\
\hline [65] & 2011 & Conference paper & Operation scheduling & Time scaling & Serial kinematics, parallel kinemaitcs \\
\hline [78] & 2013 & Journal paper & Operation scheduling & Time scaling & Serial kinematics, parallel kinemaitcs \\
\hline$[64]$ & 2013 & Conference paper & Operation scheduling & Time scaling & Industrial robotized cell \\
\hline [61] & 2011 & Conference paper & Operation scheduling & Time scaling & Serial robot (2 DoFs) \\
\hline [63] & 2013 & Journal paper & Operation scheduling & Time scaling & Industrial robotized cell \\
\hline [62] & 2012 & Conference paper & Operation scheduling & Time scaling & Industrial robotized cell \\
\hline$[88]$ & 2008 & Conference paper & Operation scheduling & Sequence scheduling & Flexible manufacturing system \\
\hline [89] & 2015 & Journal paper & Operation scheduling & Sequence scheduling & Industrial robotized cell \\
\hline$[66]$ & 2015 & Conference paper & Operation scheduling & Sequence scheduling & Industrial robotized cell \\
\hline [67] & 2017 & Journal paper & Operation scheduling & Sequence scheduling & Industrial robotized cell \\
\hline [90] & 2008 & Conference paper & Operation scheduling & Sequence scheduling & Mechatronic system (1 DoF)-elevator \\
\hline [91] & 2012 & Journal paper & Operation scheduling & Sequence scheduling & Industrial robotized cell \\
\hline [92] & 2010 & Conference paper & Operation scheduling & Sequence scheduling & Industrial robotized cell \\
\hline
\end{tabular}

PTP = Point-To-Point 


\subsection{Trajectory Optimization}

The optimization of movements can be used to reduce terms such as time, vibration content and energy consumption of mechatronic and robotic systems [93]. The path and the motion profiles are modified in order to be more energy efficient. An important aspect of this approach is the application to existing systems without modifying or redesigning any hardware. Considering the trajectory, two main different groups can be defined: the point-to-point (PTP) trajectory, also called rest-to-rest (RTR), and the multi-point $(M P)$ trajectory. Their applicability to different robotic and mechatronic systems depends on the complexity of both the kinematics and the level of detail of the considered dynamic model.

\subsubsection{Point-to-Point Trajectory Optimization}

In accord with [73], PTP trajectory optimization methods can be classified into inverse and direct approaches.

In the inverse approach [38-60], the mechatronic and robotic system is formulated through a mathematical model, generally simplified in order to reduce the computational effort. The trajectory, usually expressed as a polynomial function, is then synthesized taking into account the energy content and is minimized in an optimization.

On the contrary, the direct approach $[37,71-73,75,84,85]$ models the mechatronic and robotic system in a more complete way without neglecting the contribution of secondary loss terms (e.g., motor losses, motor-drive losses, auxiliaries losses, etc.) and taking into account also the non-ideal behavior of losses, as some of these are motion parameter-dependent. The model is then exploited to test different motion planning strategies (i.e., different pre-defined motion profiles) and to assess the impact of different parameters on the energy consumption through a sensitivity analysis.

Both the approaches have been applied to various electro-mechanical systems. In principle, they could be applied to systems with a different level of complexity and number of degrees of freedom. However, the direct approach is generally applied only on test cases with a low number of degrees of freedom: simple one-degree-of-freedom systems (e.g., systems driving a constant inertia such as an elevator [71,73], or a generic system [72]) or two-degrees-of-freedom systems (e.g., storage and retrieval vehicles [74,75]). This is because the direct approach involves a higher computational effort. However, an exception is given by the $[37,84,85]$ works, for which an extended model of a six-degrees-of-freedom anthropomorphic industrial manipulator is created, exploiting a model-based simulation tool. Indeed, the inverse approach has been adopted on a broad variety of systems with one degree of freedom-such as a liquid-crystal display glass-handling robot [43,44,46]; a toggle mechanism [45,47]; a precision positioning table [51-53]; a linear axis [57]; generic, one-degree-of-freedom systems [55,56,58]; and one-degree-of-freedom manipulators mounted on a flexible base [38,39]—with two degrees of freedom with rigid [49] and flexible links [59]; with three degrees of freedom, such as a redundant planar manipulator [40,54]; and with six degrees of freedom, such as an anthropomorphic industrial manipulator [41,42,48].

Considering the inverse approach, the mathematical model is simplified to reduce the computational effort for calculating the optimal trajectory. Usually, only the kinematic and dynamic properties (e.g., length, mass and inertia of links, gear ratios, friction coefficients, etc.) of the mechanical system and electrical parameters (e.g., motor winding resistance, motor torque and voltage constants) are taken into account and the linear behavior is modeled. Thus, the mechanical model can be expressed as

$$
\tau=\mathbf{J}(\boldsymbol{\vartheta}) \ddot{\vartheta}+\mathbf{H}(\boldsymbol{\vartheta}, \dot{\vartheta}) \dot{\vartheta}+\mathbf{G}(\boldsymbol{\vartheta}) \boldsymbol{\vartheta}
$$

where $\vartheta$ is the vector of the independent coordinates; $\tau$ is the vector of the input control torque; $\mathbf{J}(\vartheta)$ is the inertia matrix; $\mathbf{H}$ is a matrix that includes viscous friction, Coriolis and centrifugal terms; and $\mathbf{G}$ is 
a matrix that includes the gravitational and the Coulomb friction terms. The electrical equations of DC motor drivers at the $j$ th axis are usually written as follows:

$$
\left\{\begin{array}{l}
e_{j}=R_{j} i_{j}+L_{j} \frac{d i_{j}}{d t}+K_{e, j} \dot{\vartheta}_{j} \\
\tau_{j}=K_{t, j} i_{j},
\end{array}\right.
$$

where $e_{j}$ is the supply voltage; $i_{j}$ is the current flowing in the winding; $R_{j}$ and $L_{j}$ are the resistance and the inductance of the motor, respectively; $K_{t, j}$ is the motor constant, and $K_{e, j}$ is the motor velocity constant. An exception to this common way to operate is presented in [48], where the losses of servo drives and inverters are taken into account as the bus-DC sharing is considered.

After the model development, the second step of the inverse approach is the definition of the trajectory type and the parameters to be optimized, that is, design variables. Often, for serial robots, the trajectory is formulated in the joint space exploiting either a high-degree polynomial [41,43-47,51-53] or a B-spline formulation $[40,48,49]$. In [42], the phase-space representation (i.e., joint velocity versus joint angle interpolation with B-splines for each joint) of the trajectory between two points is considered, while in $[38,39,57]$ and in $[55,56,58]$, a cycloidal trajectory and a modified parabolic velocity profile are adopted, respectively. When a high-degree polynomial formulation is chosen, the joint angular displacement is expressed as

$$
\vartheta(t)=a_{0}+a_{1} t+a_{2} t^{2}+a_{3} t^{3}+\ldots+a_{n} t^{n}
$$

where each coefficient $a_{i}$ (with $i=0,1, \ldots, n ; n$ being the degree of the polynomial) is a real number and $a_{n} \neq 0$. Under this condition, $n+1$ coefficients are to be determined. In a PTP motion, the initial and final position, speed, and acceleration are usually imposed. Therefore, at least a fifth-degree polynomial should be defined to satisfy all the constraints. In order to obtain a smoother motion and to apply an optimization process, a polynomial with a degree higher with respect to the number of imposed values has to be chosen. However, increasing too greatly the degree of the polynomial does not lead to significant increment in saved energy, as shown in [46]. When a B-spline formulation of joint-space profiles for serial robots is considered for trajectory optimization, the joint position profile $\vartheta_{i}(t)$ of the $i$ th axis is expressed as

$$
\vartheta(t)=\sum_{j=0}^{m} c_{i, j} N_{j, d}\left(t, k_{i}\right), \quad \forall i=1,2, \ldots, n,
$$

where $N_{j, d}\left(t, k_{i}\right)$ are the basis functions and $c_{i, j}$ are the $m+1$ control points [48].

The next step of the approach is to define a function to be minimized, referred to as the cost or objective function. There have been several function formulations proposed in literature; these include the following:

- minimum effort (i.e., sum of squared torque) [40,59];

- minimum torque-rate (i.e., sum of squared-derivative torque) [59];

- minimum electrical energy [42-47,51-53,55,57,58], expressed as $\int_{0}^{T} e(t) i(t) d t$;

- minimum mechanical energy $[38,39,41]$, expressed as $\int_{0}^{T} \dot{\vartheta}(t) \tau(t) d t$;

- minimum of grid energy (i.e., considers the energy exchanged between axes due to recovery process) [49];

- minimum of losses in a robot DC motor drive [56].

In addition to the energy expenditure, other quantities can be minimized, such as the residual vibration amplitude [38,39].

A wide range of optimization algorithms are used to find the optimum trajectory. These include the following:

- gradient-based optimization algorithms [48], and more specifically, sequential quadratic programming (SQP) [56]; 
- genetic algorithms (GA) [41,57];

- real-coded genetic algorithms (RGA) [43-47,51-53];

- radial-basis function networks and genetic algorithms (RBF-GA) [38];

- Pontryagin's minimum algorithm (PMP) [55,58];

- metaheuristic algorithms (MA) [39];

- discrete dynamic programming (DDP) [59];

- iterative dynamic programming (DP) [42].

In the direct approach, the electromechanical model of the system is more complex compared to the previous system; in fact, in this case, the non-linearities and secondary losses contributions are taken into account. The model, besides the dynamics of the system, has to include several energy losses due to the mechanical parts, the motor and the drive system. In Table 3, a qualitative categorization of energy losses by proportionality to the speed $\dot{\vartheta}$ and torque $\tau$ is reported $[15,72,73,84,85]$.

Table 3. Qualitative categorization of energy losses [15,72,73,84].

\begin{tabular}{llll}
\hline & Loss Type & Definition of Energy & Proportionality \\
\hline \multirow{2}{*}{ Load } & Coulomb friction & $E_{\text {coulomb }}(t)=\int_{0}^{T} f_{c} \operatorname{sign}(\vartheta(t)) \vartheta(t) d t$ & $\sim \dot{\vartheta}$ \\
& Viscous damping & $E_{\text {viscous }}(t)=\int_{0}^{T} f_{v} \dot{\vartheta}(t)^{2} d t$ & $\sim \dot{\vartheta}^{2}$ \\
\hline \multirow{2}{*}{ Resistive } & $E_{R}(t)=\int_{0}^{T} R i(t)^{2} d t$ & $\sim \tau^{2}$ \\
& Core (hysteresis and eddy) & $E_{\text {core }}(t)=\int_{0}^{T} f_{\text {core }} \dot{\vartheta}(t)^{2} d t$ & $\sim \dot{\vartheta}^{2}$ \\
& Stray & $E_{\text {stray }}(t)=\int_{0}^{T} f_{\text {stray }} i(t)^{2} d t$ & $\sim \tau^{2}$ \\
& Coulomb friction & $E_{\text {coulomb }}(t)=\int_{0}^{T} f_{c} \operatorname{sign}(\vartheta(t)) \vartheta(t) d t$ & $\sim \dot{\vartheta}$ \\
& Viscous damping & $E_{\text {viscous }}(t)=\int_{0}^{T} f_{v} \dot{\vartheta}(t)^{2} d t$ & $\sim \dot{\vartheta}^{2}$ \\
& Holding brake & $E_{\text {brake }}=$ const. & const. \\
\hline \multirow{2}{*}{ Inverter } & Resistive & $E_{R}(t)=\int_{0}^{T} R i(t)^{2} d t$ & $\sim \tau^{2}$ \\
& IGBT switching & $E_{\text {sw }}=$ const. & const. \\
& PLC controller & $E_{P L C}=$ const. & const.
\end{tabular}

IGBT = Insulated Gate Bipolar Transistor PLC = Programmable Logic Controller

In this case, the trajectory is not synthesized by an optimization process, but instead, different standard pre-defined industrial motion laws are considered in order to evaluate their impact on the energy consumption:

- triangular velocity [71-73];

- harmonic [73];

- cycloidal [71-73];

- third-degree polynomial [73];

- fifth-degree polynomial [71-73];

- seventh-degree polynomial [71-73];

- modified trapezoidal [73-75];

- modified sinusoidal [73];

- Gutman 1-3 [73];

- Freudenstein 1-3 [73];

- Freudenstein 1-3-5 [73].

The trajectories are defined following three different approaches: In $[37,71,72,74,75,84,85]$, they are defined numerically with a time discretization. In [54], an analytic approach is exploited. Finally in [73], 
it is demonstrated that the energy losses in a PTP motion depend only on the root-mean-square (RMS) value of the speed or acceleration. The RMS values are obtained by multiplying some characteristic coefficients (i.e., $c_{V_{R M S}}$, and $c_{A_{R M S}}$ ) that describe the "geometry" of the profile by the parameters of the PTP motion (i.e., displacement $\vartheta$, and inverse of the cycle time $T$ ), as follows:

$$
\begin{aligned}
& \dot{\vartheta}_{R M S}=\frac{\theta}{T} c_{V_{R M S}} \\
& \ddot{\vartheta}_{R M S}=\frac{\theta}{T^{2}} c_{A_{R M S}}
\end{aligned}
$$

The direct method involves the comparison of different planning strategies on the basis of some metric related to the energy consumption. In [71], the electrical energy is considered, while in [72,74,75], the grid energy is chosen. This is done also in [73], but there it is purged from the terms that do not depend on the motion. Finally, in [54], the sum of the squared torque is used.

Because the direct method is numerical, it allows for analyzing the impact that every factor has on the energy consumption through a sensitivity analysis. In [73], the impact of both constant force (i.e., relevant increase of the Coulomb friction) and inertia (i.e., counterweight mass) are considered. In [72], a sensitivity analysis is performed considering a variation of the Coulomb and viscous friction coefficients of the mechanical inertia and of the axis displacement. The works of Paryanto et al. $[37,84,85]$ focus on the development of a modular model for industrial robots, able to analyse the power consumption and the dynamic behavior. They exploit the model to perform a sensitive analysis to investigate the effects of the robot operating parameter (i.e., payload, operating speed, and trajectory smoothness) on the energy consumption. Finally in $[74,75]$, the trajectory optimization of a storage and retrieval vehicle is considered. The impact of kinematic parameters (i.e., speeds and accelerations) on the energy consumption and on the lift times is analyzed. In addition, in [74], a method to calculate the optimal lifting starting point and the optimal velocity is presented.

The results show that the trapezoidal trajectory profile is a good trade-off for increasing the saved energy without affecting the cycle time. Indeed, the energy consumption is related to the constraints at the initial and final times: less constraints imply less energy consumption [46,71]. In this way, a five-degree polynomial trajectory consumes less than a cycloidal and a seven-degree polynomial [71,73]. Richiedei et al. [73] and Hansen et al. [72] show that a large constraint on the cycle time (i.e., a longer cycle time) gives a significant chance to obtain energy savings.

If a constant cycle time is considered, a different behavior is achieved [43-47,52,53]; it can be demonstrated that increasing the degree number of the polynomial trajectory profile leads to a decreasing of the absolute input energy. However, the use of a high-degree polynomial becomes counterproductive after a convergence point: the rate of saved-energy becomes smaller, while a more intensive computational effort is needed $[46,47]$.

Sergaki et al. [56] declares that 3-8\% can be achieved in industrial robot motor drives. Wang et al. [58] show an improvement of the $16 \%$ with respect to a conventional system. Richiedei et al. [73] show it is possible to obtain energy savings greater than $30 \%$ of the maximum possible consumption (within the feasible domain) in a non-fixed cycle time. The modified parabolic velocity profile suggested by Park et al. [55] allows savings of up to $12.5 \%$ in each repetition of the PTP motion and furthermore, up to $33 \%$ of input energy under the same peak acceleration. A comparison of methods in terms of achievable energy savings is not available and, as a result of the various and different ranges of applications, this would be hard to perform.

\subsubsection{Multi-Point Trajectory Optimization}

In MP trajectory optimization, the energy consumption of a mechatronic system is achieved by both optimizing the path and the timing [68-70,79-81]. In literature, the studied systems are usually multi-degrees-of-freedom systems. If planar motion is to be achieved, both planar (e.g., two-degrees-of-freedom [70] and three-degrees-of-freedom [68] redundant manipulators) and spatial kinematics (e.g., six-degrees-of-freedom anthropomorphic industrial manipulators [69,79-81]) are exploited. The system dynamics are usually modeled by means of a classical formulation, 
Equations (1) and (2). Generally, parameters are retrieved by datasheet, by CAD, or are estimated on the basis of some assumptions $[68,70,79-81]$. Rarely are the dynamic parameters obtained through an identification procedure such as that in [69], for which the robot performs a specific excitation trajectory and data about torque, speed and acceleration are collected directly or indirectly before the dynamic parameters are extrapolated.

Different techniques can be found in literature for the applied methodology and the trajectory representation. In [79], a path of at least three points (either "no-fly" or "fly" points), connected through straight lines and circular arcs in the proximity of a fly point is planned. The method searches among alternative paths, close to the fly points, so as to reduce the energy consumption during the execution of the trajectory, without worsening the cycle time. In [80], a similar approach is followed, that is, initial and final points and a series of intermediate points are considered, but in this case, the joint-space path is interpolated through a non-uniform cubic B-spline. In [68], a variational approach within the B-spline function is implemented. In $[69,81]$, the searching methods are used in combination with a commercial robotic programming studio. This provides a set of trajectories in the form of the "time history" of each joint and, by evaluating these, the trajectory that allows for the minimum consumption is chosen. Finally, in [70], a set of via-points in between the initial and final position, also taking into account the presence of obstacles, is searched by using an invasive weed optimization (IWO) method; then the computed points are connected with a cubic polynomial to form a continuous and relatively smooth trajectory.

This approach considers several cost functions to be minimized in the optimization process in order to retrieve the less-energy-hungry trajectory profile:

- minimum effort (i.e., sum of squared torque) [80];

- minimum electrical energy [68], expressed as $\int_{0}^{T} e(t) i(t) d t$;

- minimum mechanical energy $[70,81]$, expressed as $\int_{0}^{T} \dot{\vartheta}(t) \tau(t) d t$;

- minimum grid energy [79];

- minimum of squared velocity [69];

- minimum of acceleration average value [80].

The algorithms utilized for the searching phase are the following:

- invasive weed optimization (IWO) [70];

- artificial immune system (AIS) [80];

- steepest gradient method (SGM) [68];

- genetic algorithm (GA) [80];

- weighted objective genetic algorithm (WGA) [80].

The MP trajectory optimization method allows for optimizing the energy consumption without detrimental effects on the cycle time. In some cases, the cycle time can even be shortened. A comparison between different methods in terms of energy saved is not straightforward. Indeed, this highly depends on several parameters, such as the system type and the considered task. Some authors $[68,69,79,81]$ compare the results of their optimization algorithm with that provided by a commercial integrated development environment for robotics. Paes et al. [69] claim savings of energy of up to $4 \%$, compared to the fastest trajectory obtained with ABB RobotStudio, in a pick-and-place movement. Furthermore, the cycle time results are 3\% shorter. Mohammed et al. [81] achieve an energy reduction of up to $30 \%$ with their module compared to ABB RobotStudio in a pick-and-place task, both with and without a payload. In [79], savings of up to $18 \%$ of the energy, compared to the fastest trajectory obtained with ABB RobotStudio in a pick-and-place operation, are achieved, while the cycle time is shortened by $11 \%$. Hirakawa et al. [68] consider three kinds of operations: a linear trajectory in which up to $30 \%$ of energy is saved, a top-to-bottom linear trajectory in which up to $70 \%$ of energy is saved, and a circular trajectory that consumes $42 \%$ less energy. 


\subsection{Operations Scheduling}

Operations scheduling is a further approach related to the motion planning modification that can be found in literature, which saves energy by reducing the energy consumption of a single robotic system or a robotic cell $[61-67,76-78,88,89,91,92]$. In a robotic cell, a robot works synchronously with other robots or mechatronic systems, and the whole work has to be balanced in order to avoid queues. Moreover, in some operations, robots have to share the same working area. Thus, a robot waits for the completion of the previous robotic operation before executing its task in order to avoid collisions. The scheduling approach focuses on the optimization of the robot cell operations as a whole, and two main methods can be found: time scaling [61-65,76-78] and sequence scheduling $[66,67,88,89,91]$.

\subsubsection{Time Scaling}

This method is based on the fact that, in most of the cases, an industrial robot has been programmed to realize the operation as fast as possible (i.e., shortest cycle time without considering the energy consumption) and with a consistent idle time, or wasted time, between two operations until the next operation is run. This approach implements the idea that a sensible energy-expenditure reduction could be achieved by slowing down as much as possible the speed (i.e., reduction of acceleration mean and peak values). The consequent increment in the operation time is compensated for by speeding up the slow movements and by reducing idle times; thus the cycle time is not affected.

In $[76,77]$, this approach is applied to optimize the motion of an anthropomorphic robotic cell (electro-mechanical model is needed) from the end of a process to the home position, in order not to affect the overall industrial process. Moreover, the activation of a mechanical brake during the robot idle state (also in short-time idle state) is considered to reduce the energy consumption. The works $[65,78]$ consider the scheduling optimization of a serial and a parallel manipulator, respectively, while performing a pick-and-place task. Also in this case, the optimization can be performed only on a selection of paths in order not to affect the manufacturing process' efficiency and robustness. In [61-64], an improved method, called dynamic time scaling, is considered. This exploits dynamic programming, instead of the linear assumption adopted in the previous method, to further reduce energy consumption. Wigstrom and Lennartson [61] prove the improvements that the new method can give when considering a two-degrees-of-freedom planar manipulator. In [62-64], it is also applied to a generic robotic cell.

\subsubsection{Sequence Scheduling}

Other approaches deal with searching a more energy-efficient sequence of operations by keeping the cycle time fixed. In [88], the authors aim to reduce the acceleration load in a production cell by processing and optimizing a given working schedule, without compromising on the cycle time, and warranting the collision and deadlock avoidance. The idle time between different operations is then used to reduce velocities and accelerations for reducing the energy consumption. With the same purpose, in [91,92], the consumption of the robots into a scheduling model of the overall system is evaluated: the energy requested to perform each operation is modeled and parametrized as a function of the operation's execution time. The optimal energy schedule is then derived by solving a mixed-integer non-linear programming problem.

The goal of the sequence planning approach $[66,67]$ is to reduce the energy consumption of individual and interacting robots in a working station without changing the original paths or the total cycle time. The optimization problem is formulated as the minimization of the weighted squared angular accelerations for all joints (approximate measure of the energy consumption). The weight factor is meant to tune the optimization on the basis of the influence the acceleration of a joint has on the overall energy consumption. Constraints on the shared zone are defined when a group of robots have to move in and out of shared zones (a robot may therefore need to wait for another robot before entering the zone). This method does not rely on a dynamic model, and thus it does not require 
system identification or confidential robot data. Instead, it is based on an initial sampling of trajectories (i.e., joints displacement, velocity, and acceleration) and torque (i.e., derived from current) performed during the execution of a movement in real time. From this logged data, the optimization process retrieves an optimized trajectory in which idle times are reduced and waiting times are eliminated.

\section{Mixed Approaches}

Recently, more attention has been paid to the design and control of mechatronic and robotic systems by also considering the minimization of the consumed energy. In this regard, new techniques are being studied and validated considering both the hardware and software modification of the automatic system.

Two main ideas seem to have potential to allow the reaching of important results in this field, also overcoming the already achieved results. These rely on the co-exploitation of either elastic-energy storing systems or energy-sharing devices and optimal motion planning techniques, that is, natural motion and optimized sharing, respectively.

\subsection{Natural Motion}

This method involves the modification of the physical system by means of the insertion of elastic elements into the mechanism, with proper motion planning [13,94-98], with the aim of exploiting the free vibration mode of a mechanical system performing a cyclic task. In this way, most of the required energy needed for carrying out the task could be supplied by the elastic elements instead of the actuators (i.e., elastic elements store energy during part of the cycle as potential elastic energy, and then they release it to the rigid bodies, increasing their kinetic energy). Then, the actuators have to counteract the effect of dissipative forces and guide the mechanism through the desired trajectory. A possible drawback of this approach is when the end-effector has to be maintained in a fixed position (e.g., during a picking or a placing operation). This could be overcome by releasing the mechanical brake during the stop [94]. Two different approaches are presented in [97]:

- Natural dynamics modification (NDM), in which the mechatronic system body or parts of it are designed to perform a given periodic task efficiently; that is, the system's natural frequency is adapted to the task.

- Natural dynamics exploitation (NDE), in which the mechatronic system motion is altered in order to exploit the system's natural frequency; that is, the task is adapted to the system's characteristics.

Almost all works deal with planar mechanisms. Barreto et al. [94] consider a multibody optimization of a two-degrees-of-freedom, five-bar linkage in order to find the proper joints' spring constants as well as the trajectory that minimizes the torque requested to the motor, exploiting the natural motion. The formulation for both the NDM and NDE approaches are derived; that is, the best elastic constant and the optimal cycle frequency are computed, respectively. In [97], a two-degrees-of-freedom manipulator with prismatic joints in which elastic elements are inserted is considered. Here, an off-line analytical method for energy consumption minimization that exploits both the NDM and NDE methods is presented. In [96], a NDM approach in which a new design concept of an energy-saving planar serial robot composed by springs and reaction wheels, as actuators are considered. An enhancement can be found in [95], where adaptive elastic devices are adopted at each joint of a SCARA (Selective Compliance Assembly Robot Arm) robot to reduce the total energy in pick-and-place tasks. This solution allows for choosing the cycle time and to change the start and stop points' position, as a result of a proper tuning of the adaptive elastic devices (i.e., NDM approach). A similar approach is considered in the work of Uemura and Kawamura [98]. Here, a generic energy-saving control based on system resonance for various robotic systems (i.e., multiple degrees of freedom) performing a pick-and-place task (i.e., non-linear dynamics) is proposed. Indeed, the control method adapts the joints' stiffness in order to minimize the necessary actuators' torque, considering a periodic motion that is not necessarily harmonic (i.e., strictly sinusoidal). 


\subsection{Optimized Sharing}

The addition of energy-sharing devices to mechatronic and robotic systems allows for exploiting the available kinetic energy that instead would be wasted and in addition, for opening new scenarios for motion planning optimization. This is valid both when different systems are cooperating in a robotic cell as well as in multi-degrees-of-freedom mechanisms. Even if not many works have been published, some interesting applications and results can be found. Indeed, the concept of the cooperation between a trajectory planner and energy recovery and sharing devices has been considered $[48,49,57,72]$. However, most of these works rely only on the concept that the braking energy can be fully recovered and is then essentially subtracted from the total energy requested $[48,49,57,72]$.

On the contrary, in [86], a study of the optimal trajectory also considering the energy recoverable as the result of an optimization of the energy that could be shared between two axes working together is carried out. The results show how a proper coordination of the axes, that is, shifting and overlapping of the two trapezoidal-velocity profiles, allows for increasing the amount of utilized regenerative energy and thus the reduction of energy consumption.

In a similar manner, Hansen et al. [99] consider an optimization method of multi-axis servo-drive mechanisms able to work while the system performs its task. This method exploits the idle times that exist between the motion cycles and the energy exchange via coupled inverter DC links. Thus, the standard double-S velocity profile is stretched and delays on the starting of the motions are inserted in order to reduce the consumed energy.

\section{Conclusions}

In this paper, a comprehensive review has been provided and is therefore a reference for existing methods, techniques and technologies for enhancing the energy performance of industrial robotic and mechatronic systems. Starting from a first categorization of hardware, software and mixed techniques, different approaches and methodologies have been revised and the main results highlighted. On one hand, the hardware approaches found in the literature have been categorized into three main categories, namely, robot type selection, hardware replacement and hardware addition. On the other hand, the software approaches mainly rely on the modification and optimization of the motion planning phase and have been organized into two different groups, namely, trajectory optimization and operation scheduling. Finally, mixed approaches have been presented, including the recent techniques that exploit both hardware and software modification, showing the main contributions found in literature.

The mixed approach is currently seeing growth of novel solutions in which different combinations of hardware and software techniques drive the core of new formulations. Such a design approach offers significant potential for reducing the requested energy or enhancing the productivity in mechatronic and industrial robotic systems, as well as for opening new optimization scenarios.

Acknowledgments: This work was partially supported by the Free University of Bozen-Bolzano funds within the project TN2803: "Mech4SME3 — Mechatronics for Smart Maintenance and Energy Efficiency Enhancement".

Author Contributions: All authors have discussed and commented on the manuscript at all stages. More specifically, G.C. collected the related literature, conducted the analysis, and completed the draft writing under the supervision of R.V., E.W. and R.V. contributed to the revision of the paper structure and the presentation style, as well as the proofreading of the paper.

Conflicts of Interest: The authors declare no conflict of interest. 


\section{References}

1. Albu-Schäffer, A.; Haddadin, S.; Ott, C.; Stemmer, A.; Wimböck, T.; Hirzinger, G. The DLR lightweight robot: Design and control concepts for robots in human environments. Ind. Robot 2007, 34, 376-385.

2. Aziz, M.; Zhanibek, M.; Elsayed, A.; Abdulrazic, M.; Yahya, S.; Almurib, H.; Moghavvemi, M. Design and analysis of a proposed light weight three DOF planar industrial manipulator. In Proceedings of the IAS IEEE 52nd Annual Meeting on Industry Application Society, Portland, OR, USA, 2-6 October 2016.

3. Böhme, L.; Suchý, J. Development of the fragmented-motion-segment concept for flexible joint robots to raise energy efficiency in handling tasks. IFAC Proce. Vol. 2012, 45, 576-583.

4. Li, Y.; Bone, G. Are parallel manipulators more energy efficient? In Proceedings of the IEEE International Symposium on Computational Intelligence in Robotics and Automation, Banff, AB, Canada, 29 July-1 August 2001; pp. 41-46.

5. Glodde, A.; Afrough, M. Energy efficiency evaluation of an underactuated robot in comparison to traditional robot kinematics. Procedia CIRP 2014, 23, 127-130.

6. Hagn, U.; Nickl, M.; Jörg, S.; Passig, G.; Bahls, T.; Nothhelfer, A.; Hacker, F.; Le-Tien, L.; Albu-Schäffer, A.; Konietschke, R.; et al. The DLR MIRO: A versatile lightweight robot for surgical applications. Ind. Robot 2008, 35, 324-336.

7. Hirzinger, G.; Sporer, N.; Albu-Schäffer, A.; Hähnle, M.; Krenn, R.; Pascucci, A.; Schedl, M. DLR's torque-controlled light weight robot III - Are we reaching the technological limits now? In Proceedings of the IEEE International Conference on Robotics and Automation, Washington, DC, USA, 11-15 May 2002; Volume 2, pp. 1710-1716.

8. Izumi, T.; Li, Z.; Zhou, H. A reduction ratio for minimizing dissipated energy in a mechatronic system with a gear train. Mechatronics 2008, 18, 529-535.

9. Izumi, T.; Zhou, H.; Li, Z. Optimal design of gear ratios and offset for energy conservation of an articulated manipulator. IEEE Trans. Autom. Sci. Eng. 2009, 6, 551-557.

10. Kim, Y.J. Design of low inertia manipulator with high stiffness and strength using tension amplifying mechanisms. In Proceedings of the IEEE International Conference on Intelligent Robots and Systems, Hamburg, Germany, 28 September-2 October 2015; pp. 5850-5856.

11. Lee, G.; Sul, S.K.; Kim, J. Energy-saving method of parallel mechanism by redundant actuation. Int. J. Precis. Eng. Manuf. Green Technol. 2015, 2, 345-351.

12. Ma, S.; Hirose, S.; Yoshinada, H. Design and experiments for a coupled tendon-driven manipulator. IEEE Control Syst. 1993, 13, 30-36.

13. Pratt, G.A.; Williamson, M.M. Series elastic actuators. In Proceedings of the IEEE International Conference on Intelligent Robots and Systems, Pittsburgh, PA, USA, 5-9 August 1995; Volume 1, pp. 399-406.

14. Roos, F.; Johansson, H.; Wikander, J. Optimal selection of motor and gearhead in mechatronic applications. Mechatronics 2006, 16, 63-72.

15. Saidur, R. A review on electrical motors energy use and energy savings. Renew. Sustain. Energy Rev. 2010, 14, 877-898.

16. Yahya, S.; Moghavvemi, M.; Almurib, H. Joint torque reduction of a three dimensional redundant planar manipulator. Sensors 2012, 12, 6869-6892.

17. Yang, A.; Pu, J.; Wong, C.; Moore, P. By-pass valve control to improve energy efficiency of pneumatic drive system. Control Eng. Pract. 2009, 17, 623-628.

18. Yin, H.; Huang, S.; He, M.; Li, J. An overall structure optimization for a light-weight robotic arm. In Proceedings of the IEEE 11th Conference on Industrial Electronics and Applications, Hefei, China, 5-7 June 2016; pp. 1765-1770.

19. Ruiz, A.; Fontes, J.; Da Silva, M. The influence of kinematic redundancies in the energy efficiency of planar parallel manipulators. In Proceedings of the ASME International Mechanical Engineering Congress and Exposition, Houston, TX, USA, 13-19 November 2015.

20. Gale, S.; Eielsen, A.; Gravdahl, J. Modelling and simulation of a flywheel based energy storage system for an industrial manipulator. In Proceedings of the IEEE International Conference on Industrial Technology, Seville, Spain, 17-19 March 2015; pp. 332-337. 
21. Xu, J.; Yang, J.; Gao, J. An integrated kinetic energy recovery system for peak power transfer in 3-DOF mobile crane robot. In Proceedings of the IEEE/SICE International Symposium on System Integration, Kyoto, Japan, 20-22 December 2011; pp. 330-335.

22. Ho, T.; Ahn, K. Design and control of a closed-loop hydraulic energy-regenerative system. Autom. Constr. 2012, 22, 444-458.

23. Wang, T.; Wang, Q. An energy-saving pressure-compensated hydraulic system with electrical approach. IEEE/ASME Trans. Mechatron. 2014, 19, 570-578.

24. Luo, X.; Sun, H.; Wang, J. An energy efficient pneumatic-electrical system and control strategy development. In Proceedings of the American Control Conference, San Francisco, CA, USA, 29 June-1 July 2011; pp. 4743-4748.

25. Wang, T.; Ren, H. Reduction of power consumption for fluidic soft robots using energy recovery technique. In Proceedings of the IEEE International Conference on Information and Automation, Ningbo, China, 1-3 August 2016; pp. 1403-1408.

26. Kapoor, R.; Parveen, C. Comparative study on various KERS. Lect. Notes Eng. Comput. Sci. 2013, 3, $1969-1973$.

27. Yao, Z.; Wan, J.; Li, B.; Qian, J.; Wang, S. Research and application of elevator energy-saving devices with super capacitor to store energy. Lect. Notes Electr. Eng. 2011, 122, 429-436.

28. Wijenayake, A.; Gilmore, T.; Lukaszewski, R.; Anderson, D.; Waltersdorf, G. Modeling and analysis of shared/common DC bus operation of AC drives (Part I). In Proceedings of the IEEE Industry Applications Society Annual Meeting, New Orleans, LA, USA, 5-9 October 1997; Volume 1, pp. 599-604.

29. Meike, D.; Ribickis, L. Recuperated energy savings potential and approaches in industrial robotics. In Proceedings of the IEEE International Conference on Automation Science and Engineering, Trieste, Italy, 24-27 August 2011; pp. 299-303.

30. Meike, D.; Rankis, I. New type of power converter for common-ground DC bus sharing to increase the energy efficiency in drive systems. In Proceedings of the IEEE International Energy Conference and Exhibition, Florence, Italy, 9-12 September 2012; pp. 225-230.

31. Meike, D.; Senfelds, A.; Ribickis, L. Power converter for DC bus sharing to increase the energy efficiency in drive systems. In Proceedings of the Industrial Electronics Conference, Vienna, Austria, 10-13 November 2013; pp. 7199-7204.

32. Rankis, I.; Meike, D.; Senfelds, A. Utilization of regeneration energy in industrial robots system. Power Electr. Eng. 2013, 31, 95-100.

33. Hunt, T.; Berthelette, C.; Popovic, M. Linear One-to-Many (OTM) system. In Proceedings of the IEEE Conference on Technologies for Practical Robot Applications, Woburn, MA, USA, 22-23 April 2013.

34. Jeoung, H.; Choi, J. High efficiency energy conversion and drives of flywheel energy storage system using high temperature superconductive magnetic bearings. In Proceedings of the IEEE Conference on Power Engineering Society, Singapore, 23-27 January 2000; Volume 1, pp. 517-522.

35. Khalaf, P.; Richter, H. Parametric optimization of stored energy in robots with regenerative drive systems. In Proceedings of the IEEE/ASME International Conference on Advanced Intelligent Mechatronics, Banff, AB, Canada, 12-15 July 2016; pp. 1424-1429.

36. Richter, H.; Simon, D.; Van Den Bogert, A. Semiactive virtual control method for robots with regenerative energy-storing joints. IFAC Proc. Vol. 2014, 19, 10244-10250.

37. Paryanto; Brossog, M.; Kohl, J.; Merhof, J.; Spreng, S.; Franke, J. Energy consumption and dynamic behavior analysis of a six-axis industrial robot in an assembly system. Procedia CIRP 2014, 23, 131-136.

38. Abe, A. Minimum energy trajectory planning method for robot manipulator mounted on flexible base. In Proceedings of the 9th Asian Control Conference, Istanbul, Turkey, 23-26 June 2013.

39. Abe, A. An effective trajectory planning method for simultaneously suppressing residual vibration and energy consumption of flexible structures. Case Stud. Mech. Syst. Signal Process. 2016, 4, 19-27.

40. Ayten, K.; Sahinkaya, M.; Dumlu, A. Optimum trajectory generation for redundant/hyper-redundant manipulators. IFAC Proc. Vol. 2016, 49, 493-500.

41. Bailón, W.; Cardiel, E.; Campos, I.; Paz, A. Mechanical energy optimization in trajectory planning for six DOF robot manipulators based on eighth-degree polynomial functions and a genetic algorithm. In Proceedings of the 7th International Conference on Electrical Engineering, Computing Science and Automatic Control, Tuxtla Gutierrez, Mexico, 8-10 September 2010; pp. 446-451. 
42. Field, G.; Stepanenko, Y. Iterative dynamic programming: An approach to minimum energy trajectory planning for robotic manipulators. In Proceedings of the IEEE International Conference on Robotics and Automation, Minneapolis, MN, USA, 22-28 April 1996; Volume 3, pp. 2755-2760.

43. Fung, R.F.; Cheng, Y.H. An minimum-energy-based high-degree polynomial trajectory planning and tracking control for an LCD glass-handling robot. Int. J. Intell. Eng. Syst. 2011, 4, 1-10.

44. Fung, R.F.; Cheng, Y.H. Minimum-energy trajectory planning for an LCD glass-handling robot. In Proceedings of the 4th International Conference on Intelligent Networks and Intelligent Systems, Kunming, China, 1-3 November 2011; pp. 61-64.

45. Hsu, Y.L.; Huang, M.S.; Fung, R.F. Minimum-absolute-energy trajectory planning for a toggle mechanism driven by a PMSM. In Proceedings of the International Conference on System Science and Engineering, Dalian, Liaoning, China, 30 June-2 July 2012; pp. 193-198.

46. Fung, R.F.; Cheng, Y.H. Trajectory planning based on minimum absolute input energy for an LCD glass-handling robot. Appl. Math. Model. 2014, 38, 2837-2847.

47. Hsu, Y.L.; Huang, M.S.; Fung, R.F. Energy-saving trajectory planning for a toggle mechanism driven by a PMSM. Mechatronics 2014, 24, 23-31.

48. Hansen, C.; Oltjen, J.; Meike, D.; Ortmaier, T. Enhanced approach for energy-efficient trajectory generation of industrial robots. In Proceedings of the IEEE International Conference on Automation Science and Engineering, Seoul, Korea, 20-24 August 2012; pp. 1-7.

49. Hansen, C.; Kotlarski, J.; Ortmaier, T. Experimental validation of advanced minimum energy robot trajectory optimization. In Proceedings of the 16th International Conference on Advanced Robotics, Montevideo, Uruguay, 25-29 November 2013.

50. Ho, P.; Uchiyama, N.; Sano, S.; Sawada, K.; Kato, A.; Yonezawa, T. Simple motion trajectory generation for energy saving of industrial machines. In Proceedings of the IEEE/SICE International Symposium on System Integration, Fukuoka, Japan, 16-18 December 2012; pp. 476-480.

51. Hsu, Y.L.; Huang, M.S.; Fung, R.F. Trajectory planning and control for precision positioning table driven by a PMSM. In Proceedings of the 2nd International Conference on Intelligent Control and Information Processing, Harbin, China, 25-28 July 2011; pp. 26-31.

52. Huang, M.S.; Hsu, Y.L.; Fung, R.F. Minimum-energy point-to-point trajectory planning of a simple mechatronic system. In Proceedings of the 8th Asian Control Conference, Kaohsiung, Taiwan, 15-18 May 2011; pp. 647-652.

53. Huang, M.S.; Hsu, Y.L.; Fung, R.F. Minimum-energy point-to-point trajectory planning for a motor-toggle servomechanism. IEEE/ASME Trans. Mechatron. 2012, 17, 337-344.

54. Khoukhi, A.; Baron, L.; Balazinski, M.; Demirli, K. Fuzzy-neuro optimal time-energy control of a three degrees of freedom planar manipulator. In Proceedings of the Annual Conference of the North American Fuzzy Information Processing Society, Montreal, QC, Canada, 3-6 June 2006; pp. 247-252.

55. Park, J. Motion profile planning of repetitive point-to-point control for maximum energy conversion efficiency under acceleration conditions. Mechatronics 1996, 6, 649-663.

56. Sergaki, E.; Stavrakakis, G.; Pouliezos, A. Optimal robot speed trajectory by minimization of the actuator motor electromechanical losses. J. Intell. Robot. Syst. Theory Appl. 2002, 33, 187-207.

57. Boscariol, P.; Carabin, G.; Gasparetto, A.; Lever, N.; Vidoni, R. Energy-efficient point-to-point trajectory generation for industrial robotic machines. In Proceedings of the ECCOMAS Thematic Conference on Multibody Dynamics, Barcelona, Spain, 29 June -2 July 2015; pp. 1425-1433.

58. Wang, Y.; Zhao, Y.; Bortoff, S.; Ueda, K. A real-time energy-optimal trajectory generation method for a servomotor system. IEEE Trans. Ind. Electron. 2015, 62, 1175-1188.

59. Wilson, D.; Robinett, R., III; Eisler, G. Discrete dynamic programming for optimized path planning of flexible robots. In Proceedings of the IEEE/RSJ International Conference on Intelligent Robots and Systems, Sendai, Japan, 28 September-2 October 2004; Volume 3, pp. 2918-2923.

60. Reiter, A.; Gattringer, H.; Müller, A. Real-time computation of inexact minimum-energy trajectories using parametric sensitivities. Mech. Mach. Sci. 2018, 49, 174-182.

61. Wigstrom, O.; Lennartson, B. Energy optimization of trajectories for high level scheduling. In Proceedings of the IEEE International Conference on Automation Science and Engineering, Trieste, Italy, 24-27 August 2011; pp. 654-659. 
62. Wigstrom, O.; Sundstrom, N.; Lennartson, B. Optimization of hybrid systems with known paths. IFAC Proc. Vol. 2012, 45, 39-45.

63. Wigstrom, O.; Lennartson, B.; Vergnano, A.; Breitholtz, C. High-level scheduling of energy optimal trajectories. IEEE Trans. Autom. Sci. Eng. 2013, 10, 57-64.

64. Wigstrom, O.; Lennartson, B. Sustainable production automation-Energy optimization of robot cells. In Proceedings of the IEEE International Conference on Robotics and Automation, Karlsruhe, Germany, 6-10 May 2013; pp. 252-257.

65. Pellicciari, M.; Berselli, G.; Leali, F.; Vergnano, A. A minimal touch approach for optimizing energy efficiency in pick-and-place manipulators. In Proceedings of the IEEE 15th International Conference on Advanced Robotics: New Boundaries for Robotics, Tallinn, Estonia, 20-23 June 2011; pp. 100-105.

66. Riazi, S.; Bengtsson, K.; Wigstrom, O.; Vidarsson, E.; Lennartson, B. Energy optimization of multi-robot systems. In Proceedings of the IEEE International Conference on Automation Science and Engineering, Gothenburg, Sweden, 24-28 August 2015; pp. 1345-1350.

67. Riazi, S.; Wigstrom, O.; Bengtsson, K.; Lennartson, B. Energy and peak power optimization of time-bounded robot trajectories. IEEE Trans. Autom. Sci. Eng. 2017, 14, 646-657.

68. Hirakawa, A.R.; Kawamura, A. Trajectory planning of redundant manipulators for minimum energy consumption without matrix inversion. In Proceedings of the IEEE International Conference on Robotics and Automation, Albuquerque, NM, USA, 25-25 April 1997; Volume 3, pp. 2415-2420.

69. Paes, K.; Dewulf, W.; Vander Elst, K.; Kellens, K.; Slaets, P. Energy efficient trajectories for an industrial ABB robot. Procedia CIRP 2014, 15, 105-110.

70. Sengupta, A.; Chakraborti, T.; Konar, A.; Nagar, A. Energy efficient trajectory planning by a robot arm using invasive weed optimization technique. In Proceedings of the 3rd World Congress on Nature and Biologically Inspired Computing, Salamanca, Spain, 19-21 October 2011; pp. 311-316.

71. Chen, K.Y.; Huang, M.S.; Fung, R.F. Dynamic modelling and input-energy comparison for the elevator system. Appl. Math. Model. 2014, 38, 2037-2050.

72. Hansen, C.; Eggers, K.; Kotlarski, J.; Ortmaier, T. Task specific trajectory profile selection for energy efficient servo drive movements. In Proceedings of the 31st International Symposium on Automation and Robotics in Construction and Mining, Sydney, Australia, 9-11 July 2014; pp. 514-522.

73. Richiedei, D.; Trevisani, A. Analytical computation of the energy-efficient optimal planning in rest-to-rest motion of constant inertia systems. Mechatronics 2016, 39, 147-159.

74. Schulz, R.; Monecke, J.; Zadek, H. Der Einfluss kinematischer Parameter auf den Energiebedarf eines Regalbediengerätes. Logist. J. 2012, 8, 1-10. (In German)

75. Siegel, A.; Schulz, R.; Turek, K.; Schmidt, T.; Zadek, H. Modellierung des Energiebedarfs von Regalbediengeräten und Verschiedener Lagerbetriebsstrategien zur Reduzierung des Energiebedarfs. Logist. J. 2013, 2092, 1-18. (In German)

76. Meike, D.; Pellicciari, M.; Berselli, G.; Vergnano, A.; Ribickis, L. Increasing the energy efficiency of multi-robot production lines in the automotive industry. In Proceedings of the IEEE International Conference on Automation Science and Engineering, Seoul, Korea, 20-24 August 2012; pp. 700-705.

77. Meike, D.; Pellicciari, M.; Berselli, G. Energy efficient use of multirobot production lines in the automotive industry: Detailed system modeling and optimization. IEEE Trans. Autom. Sci. Eng. 2014, 11, 798-809.

78. Pellicciari, M.; Berselli, G.; Leali, F.; Vergnano, A. A method for reducing the energy consumption of pick-and-place industrial robots. Mechatronics 2013, 23, 326-334.

79. Fenucci, A.; Indri, M.; Romanelli, F. An off-line robot motion planning approach for the reduction of the energy consumption. In Proceedings of the IEEE International Conference on Emerging Technologies and Factory Automation, Berlin, Germany, 6-9 September 2016.

80. He, T.; Zhang, Y.; Sun, F.; Shi, X. Immune optimization based multi-objective six-DOF trajectory planning for industrial robot manipulators. In Proceedings of the World Congress on Intelligent Control and Automation, Guilin, China, 12-15 June 2016; pp. 2945-2950.

81. Mohammed, A.; Schmidt, B.; Wang, L.; Gao, L. Minimizing energy consumption for robot arm movement. Procedia CIRP 2014, 25, 400-405.

82. Hauf, D.; Kruck, J.; Paryanto.; Franke, J. Energy consumption modeling of a turning table and standardized integration into virtual commissioning. Procedia Manuf. 2017, 11, 256-264. 
83. Paryanto; Merhof, J.; Brossog, M.; Fischer, C. An integrated simulation approach to the development of assembly system components. Adv. Mater. Res. 2013, 769, 19-26.

84. Paryanto; Brossog, M.; Bornschlegl, M.; Franke, J. Reducing the energy consumption of industrial robots in manufacturing systems. Int. J. Adv. Manuf. Technol. 2015, 78, 1315-1328.

85. Paryanto; Brossog, M.; Merhof, J.; Franke, J. Mechatronic behavior analysis of a customized manufacturing cell. In Proceedings of the 7th World Conference on Mass Customization, Personalization, and Co-Creation (MCPC 2014), Aalborg, Denmark, 4-7 February 2014; Brunoe, T.D., Nielsen, K., Joergensen, K.A., Taps, S.B., Eds.; Springer: Cham, Switzerland, 2014; pp. 389-399.

86. Kaviani, A.; Hadley, B.; Mirafzal, B. Regenerative energy saving in multi-axis servo-motor-drives. In Proceedings of the IEEE Energy Conversion Congress and Exposition: Energy Conversion Innovation for a Clean Energy Future, Phoenix, AZ, USA, 17-22 September 2011; pp. 3450-3457.

87. Moreno-Valenzuela, J. Time-scaling of trajectories for point-to-point robotic tasks. ISA Trans. 2006, 45, 407-418.

88. Kobetski, A.; Fabian, M. Velocity balancing in flexible manufacturing systems. In Proceedings of the 9 th International Workshop on Discrete Event Systems, Goteborg, Sweden, 28-30 May 2008; pp. 358-363.

89. Mukund Nilakantan, J.; Huang, G.; Ponnambalam, S. An investigation on minimizing cycle time and total energy consumption in robotic assembly line systems. J. Clean. Prod. 2015, 90, 311-325.

90. Tang, H.Y.; Bao, D.; Qi, W.G.; Zhang, Y.M. Optimization of elevator group control scheduling with multi-strategy switch. In Proceedings of the 7th International Conference on Machine Learning and Cybernetics, Kunming, China, 12-15 July 2008; Volume 4, pp. 2067-2072.

91. Vergnano, A.; Thorstensson, C.; Lennartson, B.; Falkman, P.; Pellicciari, M.; Leali, F.; Biller, S. Modeling and optimization of energy consumption in cooperative multi-robot systems. IEEE Trans. Autom. Sci. Eng. 2012, 9, 423-428.

92. Vergnano, A.; Thorstensson, C.; Lennartson, B.; Falkman, P.; Pellicciari, M.; Yuan, C.; Biller, S.; Leali, F. Embedding detailed robot energy optimization into high-level scheduling. In Proceedings of the IEEE International Conference on Automation Science and Engineering, Toronto, ON, Canada, 21-24 August 2010; pp. 386-392.

93. Gasparetto, A.; Boscariol, P.; Lanzutti, A.; Vidoni, R. Path planning and trajectory planning algorithms: A general overview. Mech. Mach. Sci. 2015, 29, 3-27.

94. Barreto, J.; Schöler, F.F.; Corves, B. The concept of natural motion for pick and place operations. Mech. Mach. Sci. 2017, 46, 89-98.

95. Goya, H.; Matsusaka, K.; Uemura, M.; Nishioka, Y.; Kawamura, S. Realization of high-energy efficient pick-and-place tasks of SCARA robots by resonance. In Proceedings of the IEEE International Conference on Intelligent Robots and Systems, Vilamoura, Portugal, 7-12 October 2012; pp. 2730-2735.

96. Iwamura, M.; Imafuku, S.; Kawamoto, T.; Schiehlen, W. Design and control of an energy-saving robot using storage elements and reactionwheels. Comput. Methods Appl. Sci. 2016, 42, 277-297.

97. Shushtari, M.; Nasiri, R.; Yazdanpanah, M.; Ahmadabadi, M. Compliance and frequency optimization for energy efficiency in cyclic tasks. Robotica 2017, 1-18.

98. Uemura, M.; Kawamura, S. An energy saving control method of robot motions based on adaptive stiffness optimization-Cases of multi-frequency components. In Proceedings of the IEEE/RSJ International Conference on Intelligent Robots and Systems, Nice, France, 22-26 September 2008; pp. 551-557.

99. Hansen, C.; Eggers, K.; Kotlarski, J.; Ortmaier, T. Concurrent energy efficiency optimization of multi-axis positioning tasks. In Proceedings of the 10th IEEE Conference on Industrial Electronics and Applications, Auckland, New Zealand, 15-17 June 2015; pp. 518-525.

(C) 2017 by the authors. Licensee MDPI, Basel, Switzerland. This article is an open access article distributed under the terms and conditions of the Creative Commons Attribution (CC BY) license (http:/ / creativecommons.org/licenses/by/4.0/). 